\title{
Dehydration Behavior of Goethite Blended with Graphite by Microwave Heating
}

\author{
Youichi SAITO, ${ }^{1)}$ Keita KAWAHIRA, ${ }^{2)}$ Noboru YOSHIKAWA, ${ }^{2)}$ Hidekazu TODOROKI') and Shoji TANIGUCHI ${ }^{2)}$ \\ 1) Nippon Yakin Kogyo Co., Ltd., Technical Research Center, formerly Student, Tohoku University, 6-6-02, Aza-Aoba, Aramaki, \\ Aoba-ku, Sendai, 980-8579 Japan. \\ 2) Graduate School of Environmental Studies, Tohoku University, 6-6-02, Aza-Aoba, Aramaki, Aoba-ku, Sendai, 980-8579 Japan. \\ 3) Nippon Yakin Kogyo Co., Ltd., Technical Research Center, 4-2 Kojima-cho, Kawasaki-ku, Kawasaki, $210-8558$ Japan.
}

(Received on December 21, 2010; accepted on March 15, 2011)

\begin{abstract}
In ironmaking and steelmaking processes, dehydration of wet substances and minerals combined by $\mathrm{H}_{2} \mathrm{O}$ are often recognized to be a key to stable practices. This study aims at understanding dehydration behavior of goethite blended with graphite by microwave treatment simulating drying wet sludge and poor iron ore with combined water.

Firstly blend specimens with various conditions, powdery or pelletized, were heated by microwave apparatus. Dehydration rate of goethite increased with increasing temperature. Denser specimen of pelletized was confirmed to be less effective to heat up the whole part. It was found that surplus addition of graphite decreased efficiency in dehydration rate. Thus, it was estimated that a specimen with smaller amount of graphite, whose blend ratio of $\mathrm{FeO}(\mathrm{OH}): \mathrm{C}$ was $1: 1$, had sufficiently larger distance between graphite particles, through which microwaves could adequately penetrate into the core of the specimen. To prove this hypothesis, Network Analyzer helped evaluate penetration depth of microwave by the measurement of permittivity. The measured results showed that penetration depth decreased with increasing apparent density consistently with the heating behavior by microwave treatment. At the blend ratio of 1:2 of $\mathrm{FeO}(\mathrm{OH})$ : C, however, the specimen was estimated to behave as if it was under percolation.
\end{abstract}

KEY WORDS: microwave; goethite; graphite; decomposition; dehydration; permittivity; permeability; network analyzer.

\section{Introduction}

Dehydration technique is often recognized to be a key to a successful practice in ironmaking and steemaking processes. To begin with ironmaking process, the quality of iron ore is recently getting poorer. As widely known, some iron ores contain an appreciable amount of limonite and pisolite that have combined water as goethite $\mathrm{FeO}(\mathrm{OH})$. It eventually results in a decrease of the strength of sintered ores because many large cracks are formed during sintering. ${ }^{1,2)}$ In steelmaking process, refractory lining of a ladle has to be dried and preheated to prevent thermal shock before pouring molten steel. This operation is daily work and usually conducted by burner flames.

Further, recycling process to recover valuable metals has been focused on more than before due to the increasing requirement of development of environmentally friendly process. Importance of dehydration is not an exception in this process. Some processes are proposed and already established for recycling. Some engineers introduce RHF (rotary hearth furnace) process ${ }^{3,4}$ ) to reduce iron oxides contained in dust generated from BOF (basic oxygen furnace) and sludge generated in AP (annealing and pickling) lines, being blended with cokes as a reductant. Advantage with this process is efficient removal of $\mathrm{Zn}$ known as elements circulating internally in the steel industry. The pellets treated by the furnace, usually called as DRI (direct reduced iron), are fed into blast furnace. Before pelletizing, sludge, which is very wet and typically has approximately 50 mass $\%$ water, has to be dried by appropriate equipment such as a rotary kiln.

For recycling $\mathrm{Fe}, \mathrm{Cr}$ and $\mathrm{Ni}$ from sludge generated in stainless steel mills, smelting reduction furnace with twostage tuyeres has been established. ${ }^{5)}$ Difficulty in reduction of sludge is documented. Metals contained in the sludge exist in the form of hydroxide represented by $\mathrm{Fe}(\mathrm{OH})_{3}$. It is known that hydroxide needs more heat for reduction by $\mathrm{C}$ than $\mathrm{Fe}_{2} \mathrm{O}_{3}$.

To economically dry wet sludge, metallic heat storage materials (HSM) have been proposed as drying media most recently. ${ }^{6)}$ The idea is basically to reuse exhausted gas generated inside steel works for drying sludge. Exhaust gas with around $500^{\circ} \mathrm{C}$ is flown through the heat exchange tower with countercurrent in which HSM green balls are packed. It is mentioned that large energy is necessary for water evaporation $\left(2260 \mathrm{~kJ} / \mathrm{kg}\right.$ at $\left.100^{\circ} \mathrm{C}\right)$ and that drying process often uses a large amount of primary energy. As a result of dry, a number of benefits are brought that include volume reduction and facilitation of their afterward handling.

Trial and semi-practical application of microwave tech- 
nique has been begun for environmental techniques ${ }^{7,8)}$ and metal industries ${ }^{9-14)}$ due to the possibility to reduce $\mathrm{CO}_{2}$ emission because this process does not need fossil resources represented by oil. Microwave can be widely applied, for example, for carbo-thermal reduction to directly produce pig iron, sintering of iron ores, process of metal recycling from slags and wasted materials generated internally in steel plants, drying refractory lining and so forth in place of original equipment.

A series of investigations have been carried out by the authors' group to prove that replacement of microwave technology is available for some stages of recycling process. A preliminary trial was made to reduce $\mathrm{NiO}$ powder to metallic Ni by $\mathrm{C}^{14)}$ showing successfully recovering $\mathrm{Ni}$ with this process. First attempt ${ }^{15)}$ of microwave for actual wasted resource was performed to reduce valuable metals of $\mathrm{Fe}, \mathrm{Cr}$ and $\mathrm{Ni}$ from dried sludge blended with $\mathrm{C}$. It was reported that by microwave treatment $\mathrm{Cr}$ reduction took place at around $900^{\circ} \mathrm{C}$, that was mean temperature of the specimens, measured by a thermocouple inserted. It is apparently lower than the temperature of $1200^{\circ} \mathrm{C}$ over which $\mathrm{Cr}$ reduction thermodynamically starts. It was estimated that specimen neighbored by $\mathrm{C}$ particles partially attained high temperature over $1200^{\circ} \mathrm{C}$ because $\mathrm{C}$ can be induced by microwave. Dehydration of sludge generated in a stainless steel plant ${ }^{16)}$ was subsequently tried resulting in successful drying. The sludge was proved not to be in the form of hydroxide but to contain adhered water. Thus the treatment by microwave was carried out without $\mathrm{C}$ because water could evaporate spontaneously. It was further confirmed to be even more effective to dehydrate than by conventional electric furnace. One of the reasons why microwave manner is more beneficial is attributed to the fact that heat is directly input center of the powdery specimen while heat is input from outside by an electric furnace. This behavior observed in the previous study ${ }^{16)}$ is shown in Fig. 1 where red region is hematite formed as a result of decomposition of goethite. In fact, perfect dehydration by microwave was assured by in-situ weight measurement.

Summarizing the above survey of the previous studies, decomposition of hydroxide as well as dehydration of water is a key to establishment of economically and environmentally successful recycling process. Followed by the previous study, ${ }^{13-16)}$ this study basically aims at understanding what the optimum ratio of goethite to graphite, to which microwave induces, is to decompose goethite $\mathrm{FeO}(\mathrm{OH})$ to hematite $\mathrm{Fe}_{2} \mathrm{O}_{3}$. This trial mainly focuses on the pretreatment of poor iron ores involving limonite or pisolite as well

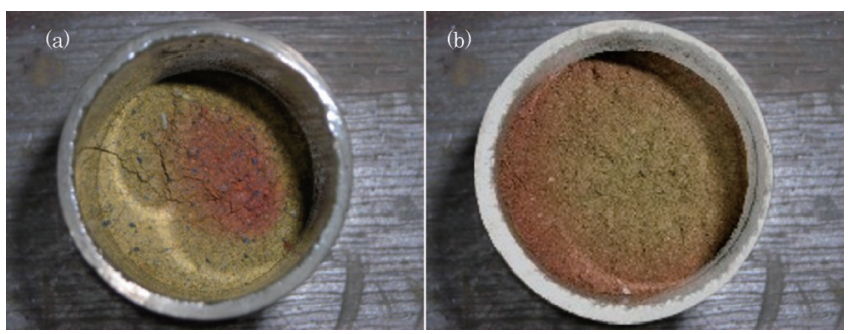

Fig. 1. Goethite powder mixed with graphite, $\mathrm{FeO}(\mathrm{OH}): \mathrm{C}=1: 2$ in molar ratio, heated by microwave (a) and heated by an electric furnace (b) whose apparent density is $0.26 \mathrm{~g} / \mathrm{cm}^{3}$. as that of sludge in which hydroxides are dominant. Permittivity ${ }^{17-19)}$ of the mixture of $\mathrm{FeO}(\mathrm{OH})$ and graphite powder has been measured in the microwave frequency at $2.45 \mathrm{GHz}$ to determine the efficiency to assess how deep microwave can penetrate with various conditions.

\section{Experimental}

\subsection{Effect of Quantity and Apparent Density on Dehy- dration of Goethite}

A mixture of goethite $\mathrm{FeO}(\mathrm{OH})$ powder (Kantokagaku Co., Ltd.) and graphite (Kojundo Chemical Laboratory Co., Ltd.: $5 \mu \mathrm{m}$ in average particle size, degree of purity is $99.7 \%$ ) powder with molar ratio of $1: 2$ was weighed. Graphite is hereinafter denoted as $\mathrm{C}$ when describing ratio to goethite. Goethite powder was preliminarily held at $180^{\circ} \mathrm{C}$ for 90 minute to remove free water. The mixture contained in a quartz crucible was set into a microwave apparatus. Three experiments were conducted with the condi-

Table 1. Experimental conditions

\begin{tabular}{cccc}
\hline Exp. No. & Diameter $(\mathrm{mm})$ & Weight $(\mathrm{g})$ & Apparent density $\left(\mathrm{g} / \mathrm{cm}^{3}\right)$ \\
\hline 1 & 28 & 4 & 0.26 \\
2 & 42 & 20 & 0.26 \\
3 & 30 & 20 & 0.65 \\
\hline
\end{tabular}

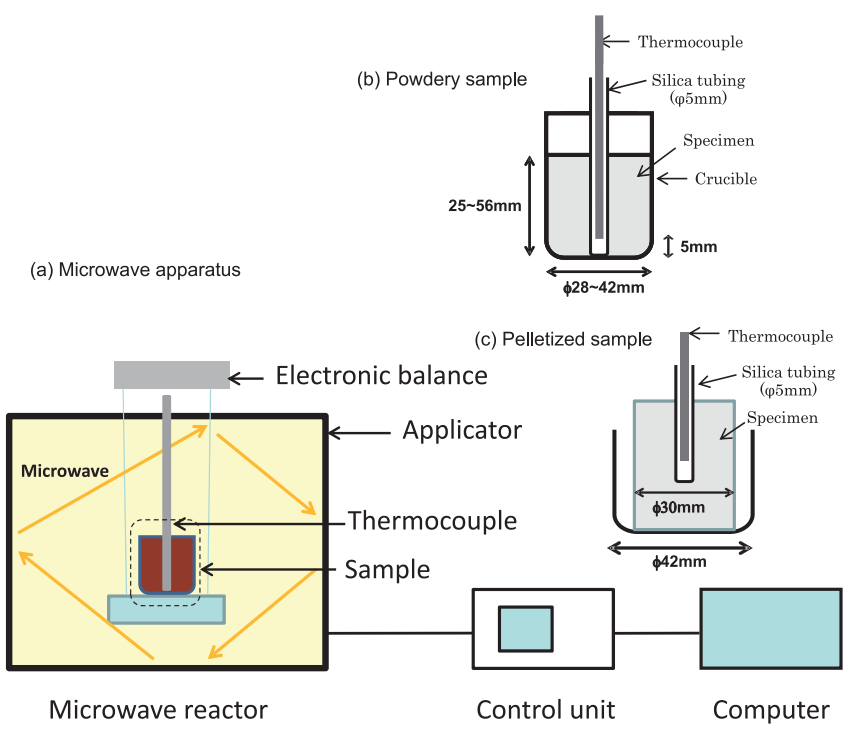

Fig. 2. Schematic of microwave apparatus (a) with set-up of powdery sample (b) and pelletized sample (c).

\section{Applicator}

$(1.5 \mathrm{~kW} \times 4)$

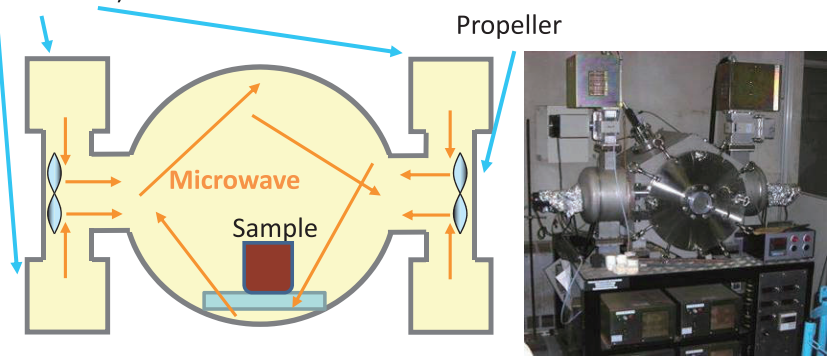

Fig. 3. Schematic and picture of $6 \mathrm{~kW}$ microwave applicator. 
tions listed in Table 1. The diameters of the quartz crucibles were chosen as 28 and $42 \mathrm{~mm}$. The weights of the mixture were 4 and $20 \mathrm{~g}$ for smaller and larger quantities, respectively. Apparent densities were adjusted as $0.26 \mathrm{~g} / \mathrm{cm}^{3}$ and $0.65 \mathrm{~g} / \mathrm{cm}^{3}$. The formers of Exp. No.1 and 2 were experimented as they were powdery specimens. Meanwhile, a pressed pellet was prepared for the latter of Exp. No.3. Multimode applicator (Shikoku Keisoku, max power $0.67 \mathrm{~kW}$, wave-frequency $2.45 \mathrm{GHz}$ ) was used for the microwave apparatus shown in Fig. 2. Basically the set-up of the specimen contained in the crucible was the same as in the previous study. ${ }^{16)}$ Thus the detailed explanation is omitted here. The specimens were heated from a room temperature to the aimed temperatures of $200,300,400$ and $500^{\circ} \mathrm{C}$ controlled by PID flowing nitrogen gas with $500 \mathrm{~mL} /$ minute. Heat treatments were carried on for 1200 seconds in which initial heating up to the aimed temperatures were included. The initial heating rate was chosen as $100^{\circ} \mathrm{C} /$ minute.

After the microwave experiments, XRD (Rad-C, Rigaku) analysis was carried out to identify the consisting phases of the specimens to examine how goethite changed. Specimens with and without graphite were examined with TG (Seiko Instruments Inc. Exstar6000, TG/DTA6300) analysis in order to compare the behaviors of weight loss from room temperature to high temperature $\left(1200^{\circ} \mathrm{C}\right)$. The condition was that initial sample weight was approximately $4.5 \mathrm{mg}$ and blank alumina pan was used for reference substance. The heating rate was adjusted as $30^{\circ} \mathrm{C} / \mathrm{min}$.

\subsection{Effect of Graphite Ratio on Heating Behavior}

The pellets $(\mathrm{FeO}(\mathrm{OH}): \mathrm{C}=1: 1$ and $1: 2$ in mole) of the same size as Exp. No.3 in Table 1 were heated by microwave to see how the graphite ratio affected on the heating behavior. The experiments were performed with an apparatus of multimode applicator (Nihon Koshuha, max power $6 \mathrm{~kW}$, wavefrequency $2.45 \mathrm{GHz}$ ) which has larger power of $6 \mathrm{~kW}$ in maximum. The apparatus is schematically shown in Fig. 3 along with a picture. The experiments were undertaken for $1,2,4$ and 8 minutes at the condition of the ratio of $\mathrm{FeO}(\mathrm{OH}): \mathrm{C}=1: 2$. The conditions of $1: 1$ and $1: 2$ were compared with the condition of heating period of 1 minute.

\subsection{Determination of Penetration Depth of Microwave}

Relative permittivity ( $\varepsilon^{\prime}$ and $\left.\varepsilon^{\prime \prime}\right)$ was measured in the conditions listed in Table 2 by means of Network Analyzer. ${ }^{18,19)}$ The molar ratios of $\mathrm{FeO}(\mathrm{OH}): \mathrm{C}$ were chosen to be $1: 1$ and $1: 2$. The sample weight was also varied at the molar ratio of $1: 1$ to determine the effect of apparent density. The powdery specimen was carefully packed in the holder not to be hollowed. In fact, less dense condition with smaller amount of packing was difficult to make due to very careful packing technique.

\section{Results and Discussion}

\subsection{Effect of Quantity and Apparent Density on Dehy- dration of Goethite}

Figures 4 to 6 show the weight losses of the specimens under the conditions listed in Table 1. The dashed line in every figure corresponds to the weight loss when goethite is completely dehydrated accounting for stoichiometry. It is assumed that the mass of graphite does not vary with dehydration. One can see that the dehydration behavior differs depending on each condition. In Exp. No.1 shown in Fig. 4, complete dehydration could not be achieved below $300^{\circ} \mathrm{C}$ while the specimen held at $400^{\circ} \mathrm{C}$ was completely dehydrated at the end. Meanwhile, the specimen held at $500^{\circ} \mathrm{C}$ was mostly decomposed after $300 \mathrm{sec}$. In fact, this tendency is

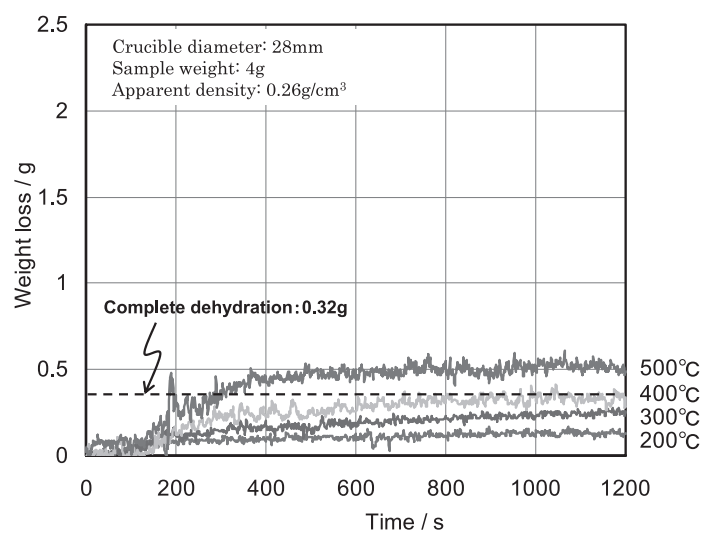

Fig. 4. Weight loss of Exp. No.1.

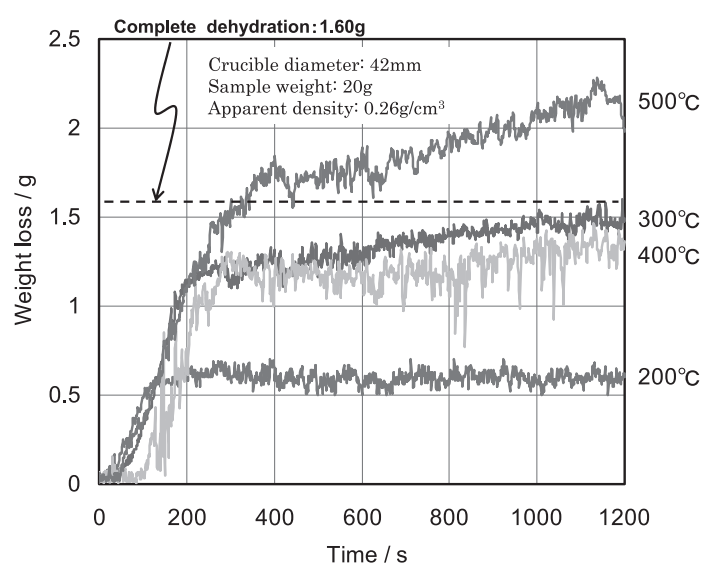

Fig. 5. Weight loss of Exp. No.2.

Table 2. Measurement condition of permittivity and penetration depth.

\begin{tabular}{ccccccc}
\hline $\begin{array}{c}\mathrm{FeO}(\mathrm{OH}): \mathrm{C} \\
(\text { molar ratio })\end{array}$ & Sample weight $(\mathrm{g})$ & $\begin{array}{c}\text { Apparent } \\
\text { density }\left(\mathrm{g} / \mathrm{cm}^{3}\right)\end{array}$ & $\mathcal{E}_{r}{ }^{\prime}$ & $\mathcal{E}_{r}{ }^{\prime}$ & $\begin{array}{c}\text { Penetration } \\
\text { depth }(\mathrm{mm})\end{array}$ & Remarks \\
\hline & 0.0687 & 0.73 & 8.84 & 3.15 & 37.35 \\
$1: 1$ & 0.1000 & 1.06 & 25.9 & 27.2 & 8.07 \\
& 0.1061 & 1.13 & 27.0 & 28.9 & 7.78 \\
\hline $1: 2$ & 0.1183 & 1.26 & 32.2 & 53.1 & 5.04 & conductive \\
\hline
\end{tabular}


in good agreement with the previous dehydration behavior ${ }^{16)}$ since the condition is mostly the same. The change in color of the specimens is in accordance to this weight loss; red region is getting larger with increasing holding temperature. As confirmed previously, ${ }^{16)}$ substance of the red color was identified as hematite while right brown, original color, as goethite by XRD analysis. It should be noted that the goethite remained without decomposed has surrounded red region same as in Fig. 1(a), except for the experiment at $500^{\circ} \mathrm{C}$ whose color was darker red rather than red. XRD analysis showed the formation of a small amount of magnetite which was estimated to make color darker. Further confirmation of magnetite formation was made using TG technique explained in detail later.

On the other hand, for Exp. No.2 shown in Fig. 5, the weight loss held at $200^{\circ} \mathrm{C}$ is obviously below the complete decomposition line while the weight losses at 300 and $400^{\circ} \mathrm{C}$ are larger indicating almost complete decomposition at the ends. The weight loss at $500^{\circ} \mathrm{C}$ is obviously above the complete dehydration which has been reached at $300 \mathrm{sec}$. with subsequent weight loss. The appearance after the experiment was the same as in Fig. 1(a) except for the experiment at $500^{\circ} \mathrm{C}$, whose color was darker red rather than red similarly to the result of $500^{\circ} \mathrm{C}$ of Exp. No.1. XRD analysis showed the formation of a small amount of magnetite which was estimated to make color darker. The tendencies of Exp. No.1 and 2 are quite similar because the dehydration behaviors are the same as in Fig. 1(a). The slight difference is the faster weight loss seen at the first 100 sec. in Exp. No. 2 whose sample weight is larger than No.1. This reason may be attributed to the fact that the absolute amount of $\mathrm{C}$ particles is larger in Exp. No.2 resulting in the quicker response in the beginning.

The behavior of Exp. No.3 using a pellet shown in Fig. 6 is totally different from the above two cases; the weight loss rates are even lower at 400 and $500^{\circ} \mathrm{C}$. The weight losses of 400 and $500^{\circ} \mathrm{C}$ have attained the complete dehydration line at the ends of experiments at last. Further, the weight losses at 200 and $300^{\circ} \mathrm{C}$ are much lower than in the case of Exp. No.2 even though the initial weight is the same. The appearance of every specimen of Exp. No.3 after the experiment was unexpectedly opposite to the Exp. No.1 and 2; the red color surrounded right brown. This implied that heat was input from the outside of the specimens.

As explained above, the color of the specimens of Exp. No. 2 tuned darker red when especially held at $500^{\circ} \mathrm{C}$. This may imply the formation of magnetite due to further reduction of hematite by graphite. Thus TG analysis was conducted to verify how reduction process proceeded. Figures 7(a) and 7(b) show the results of weight change, where minus means loss, of goethite powder without and with graphite, respectively. Obvious difference is seen in that weight loss starts at around $200^{\circ} \mathrm{C}$ and once stops at around $300^{\circ} \mathrm{C}$ followed by very gradual loss for both cases. The significant drop between 200 and $300^{\circ} \mathrm{C}$ is attributed to the decomposition of goethite according to the following reaction $^{16)}$ :

$2 \mathrm{FeO}(\mathrm{OH})(\mathrm{s}) \rightarrow \mathrm{Fe}_{2} \mathrm{O}_{3}(\mathrm{~s})+\mathrm{H}_{2} \mathrm{O}(\mathrm{g}) \uparrow \quad \Delta \mathrm{G}^{\circ}<0 \quad \mathrm{~T}>192^{\circ} \mathrm{C}$

According to the above reaction decomposition thermo-

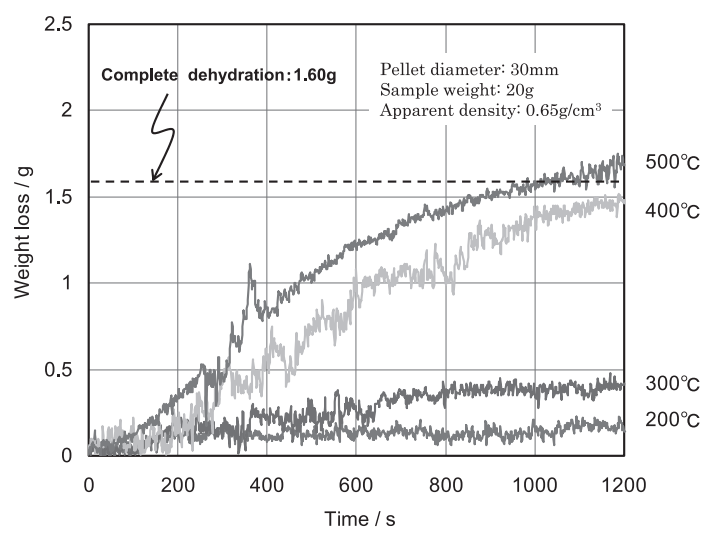

Fig. 6. Weight loss of Exp. No.3.

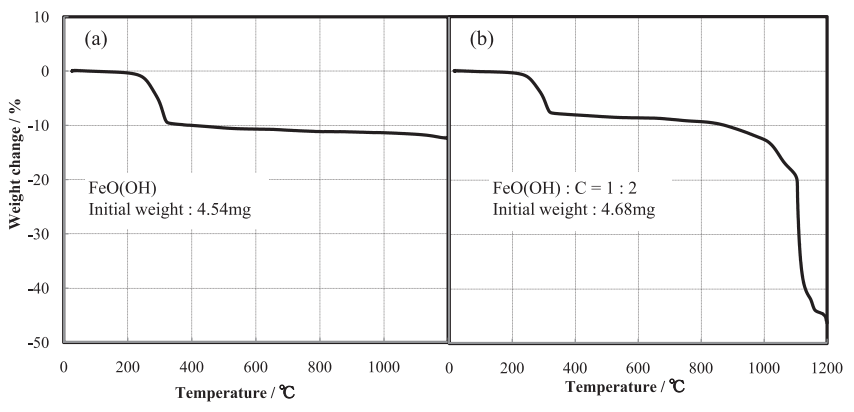

Fig. 7. Weight change of the specimens of only goethite (a) and mixture of goethite with $\mathrm{C}(\mathrm{b})$.

dynamically starts at $192^{\circ} \mathrm{C}$ that well agrees to the TG analysis. Gradual decrease in weight occurring over $300^{\circ} \mathrm{C}$ should be attributed to the vaporization of water strongly adhered on the hematite particles. ${ }^{20)}$ The line in Fig. 7(b) has more complicated behavior than that in Fig. 7(a) because of further reduction of hematite by graphite. In particular, subsequent weight loss is obviously seen at $1100^{\circ} \mathrm{C}$ in Fig. 7(b). Considering the mass balance this sharp drop corresponds to reduction of wustite to metallic Fe. One can see that relatively gradual decrease in weight between 800 and $1100^{\circ} \mathrm{C}$. This may correspond to the reduction of hematite to magnetite which further is reduced to wustite. This result tells us that the weight losses over the complete dehydration seen in the experiments held at $500^{\circ} \mathrm{C}$ in Fig. 5 are caused by this reduction by graphite. Thus it is possible for these specimens to be heated up partially ranging approximately from 800 to $1000^{\circ} \mathrm{C}$. Further, this result is consistent with the XRD peaks indicating the formation of magnetite. The reason why it was estimated not to attain over $1100^{\circ} \mathrm{C}$ is attributed to the fact that the corresponding specimens did not contain metallic iron.

\subsection{Effect of Graphite Ratio on Heating Behavior}

As mentioned above, the unexpected heating behavior was observed in Fig. 6. Specimen was heated up starting from outside unlike Fig. 1(a). The condition of Exp. No.3 was that blend ratio was $\mathrm{FeO}(\mathrm{OH}): \mathrm{C}=1: 2$ in mole with pelletized to be denser than the No.1 and 2 as listed in Table 1. Therefore, shorter distance between graphite particles due to higher apparent density was assumed to cause this difference.

This probability was then tried to be determined by the 


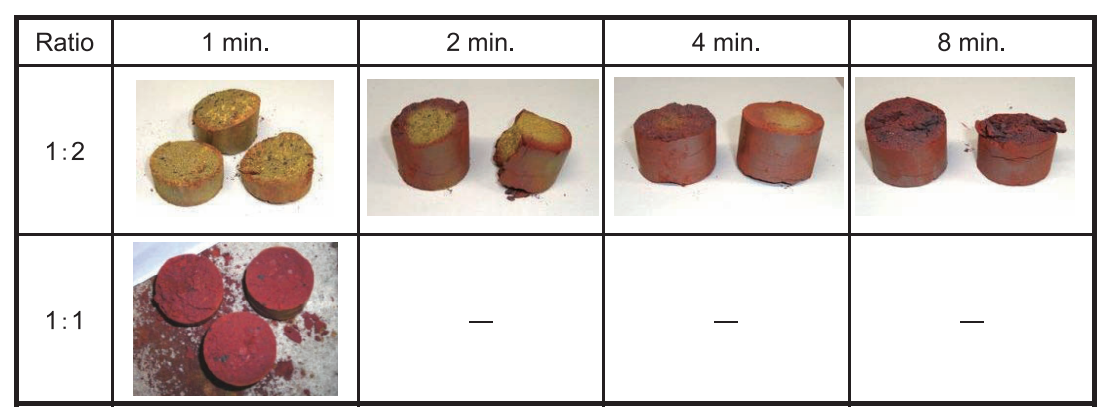

Fig. 8. Pellets $(\phi 30 \mathrm{~mm})$ of goethite blended by $\mathrm{C}$ with the different ratios $(\mathrm{FeO}(\mathrm{OH})$ :C) after treatment of $6 \mathrm{~kW}$ microwave applicator.

pelletized conditions with varying graphite ratio. Figure 8 shows appearances of the specimens after the microwave treatments by means of $6 \mathrm{~kW}$ applicator. Obviously the specimens of $\mathrm{FeO}(\mathrm{OH}): \mathrm{C}=1: 2$ show the same behavior as Exp. No.3. The reproducibility of the experiments was also confirmed owing to gradual heating from outside toward inside reaching the complete dehydration as long as for 8 minutes. However, the specimens of $\mathrm{FeO}(\mathrm{OH}): \mathrm{C}=1: 1$ have completed the dehydration reaction merely at 1 minute definitely proved by the uniformity of the red color. Consequently, it has been revealed that too much addition of graphite results in the less efficiency to heat up the specimen leading to lowering the reaction rate. Alternatively, if the denser condition is unbeneficial for dehydration, the probable reason is caused by slower transfer of water vapor from the core to the surface occasionally leading to bursting. However, such phenomena caused by the abrupt reaction have not been observed. This is why the difference in blend ratio is the cause of the higher rate of dehydration at $1: 1$.

The following mechanism is hence estimated accounting for the results mentioned above. As illustrated in Fig. 9, a specimen with smaller amount of graphite has sufficiently larger distance between graphite particles, through which microwaves can adequately penetrate into the core of the specimen. A specimen with larger amount of graphite has whereas smaller distance between graphite particles oppositely resulting in the reflection of microwave. The latter case may be getting closer to the behavior only of graphite. In Exp. No.3 of the pellet, thus microwave could heat up only surface area without advancing dehydration rate. Therefore, consecutive experiments were planned to examine how specimens behaved varying the ratio of $\mathrm{FeO}(\mathrm{OH}): \mathrm{C}$ along with apparent density by means of Network Analyzer.

\subsection{Relative Permittivity and Penetration Depth of Microwave}

Table 2 shows the measurement conditions and the obtained results. Penetration depth of microwave can be calculated by the following equation ${ }^{21)}$ :

$$
\delta=\frac{\sqrt{2} * \mathrm{c}}{\omega\left[\sqrt{\left(\varepsilon^{\prime}{ }_{r}\right)^{2}+\left(\varepsilon_{r}^{\prime \prime}\right)^{2}}-\varepsilon^{\prime}{ }_{r}\right]^{\frac{1}{2}}}
$$

where $\delta$ shows penetration depth, c the velocity of light and $\omega$ angular frequency while $\varepsilon_{r}$ ' and $\varepsilon_{r}$ " show real and imaginary parts of relative permittivity, respectively. The velocity



Fig. 9. Hypothesis of how the behavior differs between the pellets with smaller and larger amounts of $\mathrm{C}$.

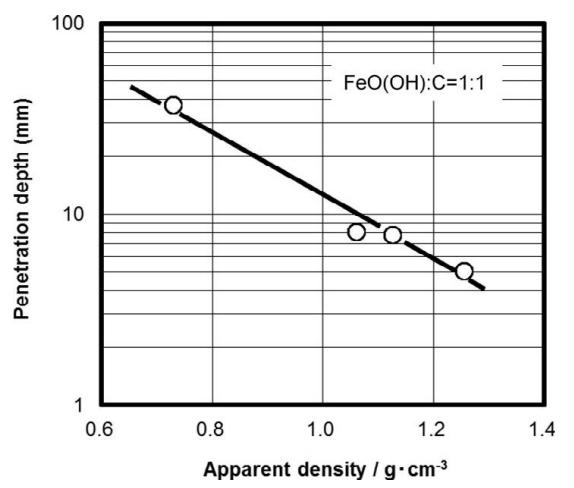

Fig. 10. Effect of apparent density on penetration depth.

of light has the following relation well-known as Maxwell's equation:

$$
\mathrm{c}=\frac{1}{\sqrt{\varepsilon_{0} \mu_{0}}}
$$

where $\varepsilon_{o}$ and $\mu_{o}$ show the permittivity and permeability under vacuum, respectively.

Figure 10 shows the logarithm of penetration depth plotted against apparent density at the blend ratio of $\mathrm{FeO}(\mathrm{OH}): \mathrm{C}=1: 1$. Clearly, the penetration depth decreases with increasing apparent density. This fact proves that microwave can appropriately penetrate toward inside if the distance between graphite particles is long enough. In contrast, calculation was impossible for the blend ratio of $1: 2$ because of the negative and quite large values in $\varepsilon_{r}$ ' and $\varepsilon_{r}$ ", respectively. Meanwhile, the values in $\mathcal{E}_{r}$ ' and $\varepsilon_{r}$ " for $\mathrm{FeO}(\mathrm{OH})$ powder without blending $\mathrm{C}$ were measured to understand its absorbing ability of microwave. The values in $\mathcal{E}_{r}$ ' and $\mathcal{\varepsilon}_{r}$ " were 2.21 and 0.03 , respectively, under the con- 
dition of sample weight of $0.02030 \mathrm{~g}$ with $0.81 \mathrm{~g} / \mathrm{cm}^{3}$ in apparent density. The value of $\mathcal{E}_{r}$ " is obviously close to zero proving that the ability of $\mathrm{FeO}(\mathrm{OH})$ to absorb microwave can be ignored. Therefore it has been proved that surplus addition of graphite results in obstructing the penetration of microwave due to too much population of graphite. This phenomenon, that the specimens behave as if they are conductive, is estimated as percolation. ${ }^{22)}$ Further measurements are necessary to fully prove this estimation. This will be discussed in a separate paper.

The optimum condition to decompose goethite has to be discussed with the obtained data. Taking into account the condition of the present study heating up the pellets with diameter of $30 \mathrm{~mm}$, at least $15 \mathrm{~mm}$ of penetration depth is required to allow microwave to penetrate into the center. The blend ratio of $1: 1$ with the apparent density of $0.73 \mathrm{~g} / \mathrm{cm}^{3}$ has $37.35 \mathrm{~mm}$ depth satisfying the critical value as realized in Table 2. Actually this condition is consistently quite similar to that of $30 \mathrm{~mm}$ diameter with the blend ratio of $1: 1$ with the apparent density of $0.65 \mathrm{~g} / \mathrm{cm}^{3}$ as shown in Fig. 8 .

In the present study, the blend ratio lower than 1 , such as $\mathrm{FeO}(\mathrm{OH}): \mathrm{C}=1: 0.5$, has not been tried. If the decomposition of the goethite to hematite is only focused on, the corresponding conditions should be experimented. However, our goal is not only to dehydrate the poor mineral containing combined $\mathrm{H}_{2} \mathrm{O}$ but also to reduce valuable elements along with sintering concurrently proceeding. The pellets should be strong enough not to be broken when handling afterwards. To accomplish this goal, graphite ratio has to be at least $1: 1$ by which reduction of iron oxide is available.

\section{Conclusions}

Experiments were undertaken to determine how to effectively decompose goethite with various conditions. Further penetration depth of microwave was evaluated by help of Network Analyzer by which permittivity was measured. The following words summarize the present study.

(1) Dehydration rate of goethite powder blended with graphite increased with increasing temperature in the experimental conditions between 100 and $500^{\circ} \mathrm{C}$.

(2) Denser condition, that the specimen was pelletized, was confirmed to be less effective for heating the whole part.

(3) It was found that surplus addition of graphite caused less efficiency in dehydration rate.

(4) It was estimated that a specimen with smaller amount of graphite had sufficiently wider opening of graphite particles between which microwaves could adequately penetrate into the core of the specimen.

(5) Penetration depth evaluated by Network Analyzer decreased with increasing apparent density consistently with the tendency heated by microwave.

(6) At the blend ratio of $\mathrm{FeO}(\mathrm{OH}): \mathrm{C}=1: 2$, the specimen was estimated to behave as percolation.

\section{Acknowledgement}

This study was supported by the Grant-in-Aid of Ministry of Education, Sports, Culture, Science and Technology, Japan, Priority Area on Science and Technology of Microwave- Induced, Thermally Non-Equilibrium Reaction Field (2006-10).

\section{REFERENCES}

1) T. Maeda, K. Nishioka and M. Shimizu: ISIJ Int., 49 (2009), 625.

2) H. Ono, Y. Dohi, Y. Arikata and T. Usui: ISIJ Int., 49 (2009), 722.

3) H. Oda, T. Ibaraki and Y. Abe: Nippon Steel Tech. Rep., 384 (2006), 134.

4) H. Tsutsumi, S. Yoshida and M. Tetsumoto: Kobe Steel Eng. Rep., 60 (2010), 36

5) T. Uchiyama, Y. Hara, K. Takeda, H. Uesugi and T. Nakamura: Tetsu-to-Hagané, 89 (2003), 552.

6) N. Hayashi, E. Kasai and T. Nakamura: ISIJ Int., 50 (2010), 1282.

7) D. A. Jones, T. P. Lelyveld, S. D. Mavrofield, S. W. Kingman and N. J. Miles: Resour. Conserv. Recyc., 34 (2002), 75.

8) J. A. Menendez, M. Inguanzo and J. J. Pis: Water Res., 36 (2002), 3261.

9) K. Nagata: Kinzoku, AGNE, Tokyo, 76 (2006), 870.

10) S. J. Tae, T. Tanaka and K. Morita: ISIJ Int., 49 (2009), 1259.

11) C. A. Pickles: Miner. Eng., 22 (2009), 1112.

12) W. Ren, G. Qiu, M. Hu, D. Chen, L. Wen and C. Bai: Abstracts of Int. Symp. on MW and Process. in Iron and Steelmaking and Env. Protection, The 19th Committee of JSPS, Tokyo, (2006), 22.

13) N. Yoshikawa, E. Ishizuka, K. Mashiko, Y. Chen and S. Taniguchi: ISIJ Int., 47 (2007), 523.

14) N. Yoshikawa, E. Ishizuka, K. Mashiko and S. Taniguchi: Metall. Mater. Trans. B, 38B (2007), 863.

15) N. Yoshikawa, K. Mashiko, Y. Sasaki, S. Taniguchi and H. Todoroki: ISIJ Int., 48 (2008), 690.

16) K. Iwasaki, K. Mashiko, Y. Saito, N. Yoshikawa, H. Todoroki and S. Taniguchi: ISIJ Int., 49 (2009), 596.

17) A. von Hippel: Dielectrics and Waves, John Wiley \& Sons, Inc, New York, USA, (1954).

18) M. Hotta, M. Hayashi, A. Nishikata and K. Nagata: ISIJ Int., 49 (2009), 1443.

19) M. Hotta, M. Hayashi and K. Nagata: ISIJ Int., 50 (2010), 1514.

20) C. A. Pickles: Miner. Eng., 17 (2004), 774

21) A. C. Metaxas and R. J. Meredith: Industrial Microwave Heating, Peter Peregrinus Ltd. London, UK, (1983).

22) H. Leuenberger: Adv. Powder Technol., 10 (1999), 323. 